\title{
In-line Holography with Single Atom Sensitivity: Challenges and Achievements
}

\author{
Christian Kisielowski ${ }^{1}$, Petra Specht ${ }^{2}$, Jinhui Yang ${ }^{3}$, Ian D. Sharp ${ }^{3}$
}

1. Molecular Foundry, Lawrence Berkeley National Laboratory, Berkeley CA 94720, USA

2. Dep. of Materials Science and Engineering, University of California, Berkeley, CA 94720, USA

3. Joint Center for Artificial Photosynthesis, Lawrence Berkeley National Laboratory, Berkeley 94720 , USA

Exploring the abilities of aberration-corrected electron microscopy, it is now established that single atoms can reliably be detected across the Periodic Table of elements. This capability enables electron tomography from single projections [1], spectroscopy studies of catalytically active edge sites in 2Dmaterials [2] or even the observation of single atom dynamics [3] to name a few new and exciting applications. However, the detection of single atoms comes with a "price tag": Unlike images from atom columns that can be captured with varying electron doses and signal-to-noise $(\mathrm{S} / \mathrm{N})$ ratios (Figure 1a), the contrast from a single atom can only be visualized if the total electron dose is large $\left(10^{3}-10^{5}\right.$ electrons $/ \AA^{2}$, Figure $1 \mathrm{~b}$ ) even if a $\mathrm{S} / \mathrm{N}$ ratio of only 2 is desired. Absolute values depend on the efficiency of the electron detection modes and the atomic number $Z$ of an element (Figure 1c,d) [4]. It has become accepted that the related high beam currents in the range of $1 \mathrm{~A} / \mathrm{cm}^{2}-100 \mathrm{~A} / \mathrm{cm}^{2}$ may allow maintaining bulk material properties if acceleration voltages are suitably varied between $60 \mathrm{kV}$ and 300 $\mathrm{kV}$. Nevertheless, the probing radiation still actively alters the genuine structure of surfaces, interfaces and small atom clusters because unavoidable beam-sample interactions remain relevant if binding energies are lowered compared to bulk values and the onset of atom displacements is soft without exhibiting a pronounced threshold. In atomic resolution images with single atom sensitivity such alterations are readily visible [5].

As heterogeneous materials scale below 5-10 nm, interfaces and small atom clusters allow tailoring unprecedented materials properties and it becomes essential to explicitly address their sensitivity to any probing radiation. For a detection of single atoms in these circumstances, we recently introduced low dose-rate in-line holography [3 - 5], which allows operating electron microscopes with dose rates that are comparable with best practices in biological applications while achieving single atom sensitivity by the acquisition of large image series. In this manner, structural materials integrity can be better maintained to an unexplored end. This talk highlights recent achievements including the radiation sensitivity of catalytic materials where the occurrence of an amorphous phase is radiation induced [6] and an Oswald ripening of $3 \mathrm{~nm}$ large catalysts that is stimulated by the electron beam (Figure 2) [7].

\section{References:}

[1] F-R. Chen et al, Nature Comm., DOI: 10.1038/ncomms10603, online February 18th (2016).

[2] Y. Zhu et al, Angew. Chem. Int. Ed. 53 (2014), p. 10723.

[3] C. Kisielowski et al, Phys Rev. B 88 (2013), p. 024305.

[4] G.A. Botton et al. Micron 68 (2015), p. 140.

[5] C. Kisielowski, Advanced Materials 27 (2015), p. 5838.

[6] J. A. Haber et al., Adv. Energy Mater. 5 (2015), p. 1402307.

[7] Work at the Molecular Foundry is supported by the Office of Science, the Office of Basic Energy Sciences, the U.S. Department of Energy under Contract No. DE-AC02-05CH11231. 


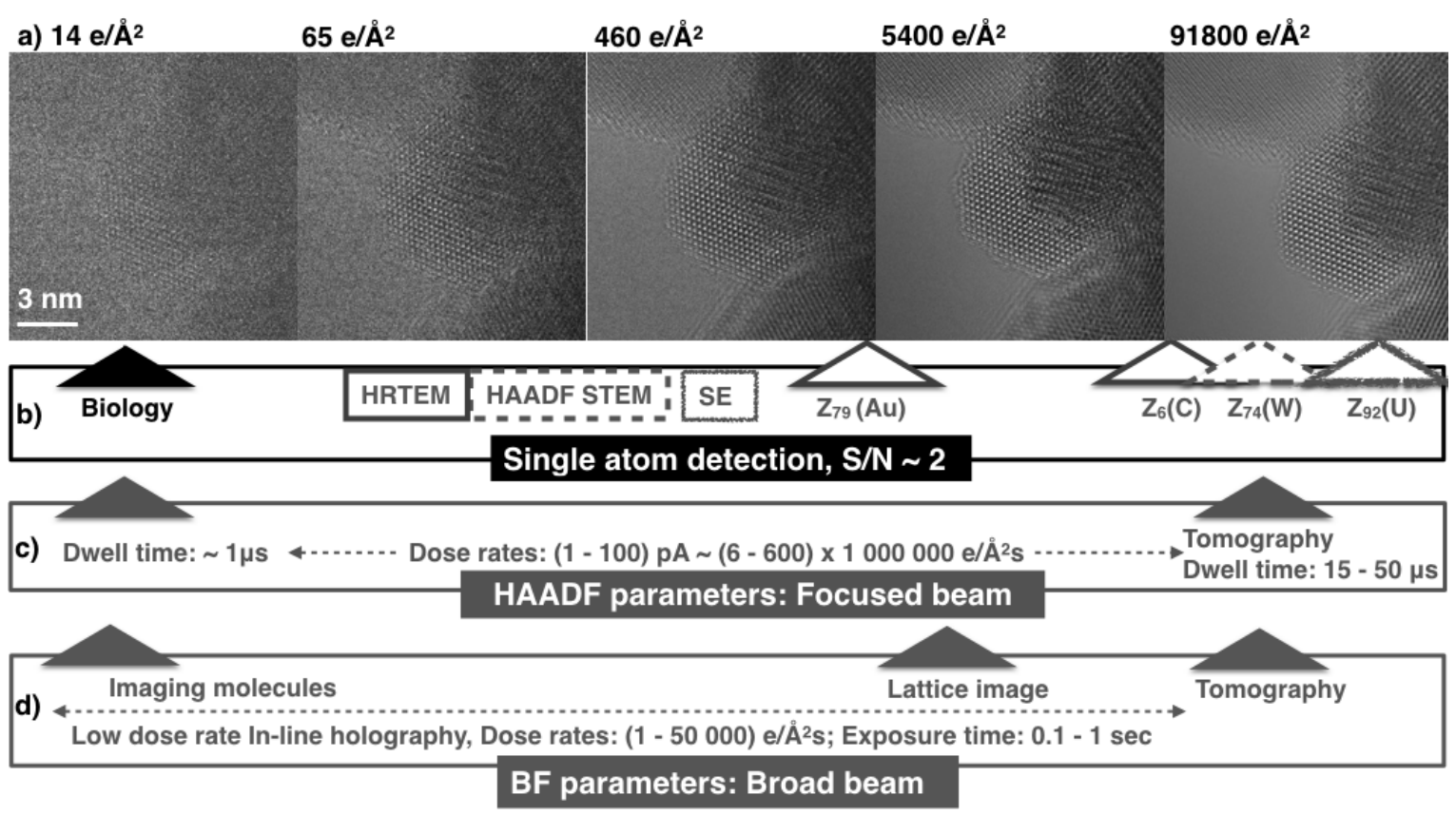

Figure 1. a) Single images of $\mathrm{Co}_{3} \mathrm{O}_{4}$ spinel using an increasing electron dose; b) required total dose to detect single atoms; c,d) typical detection parameters for the most common detection modes.

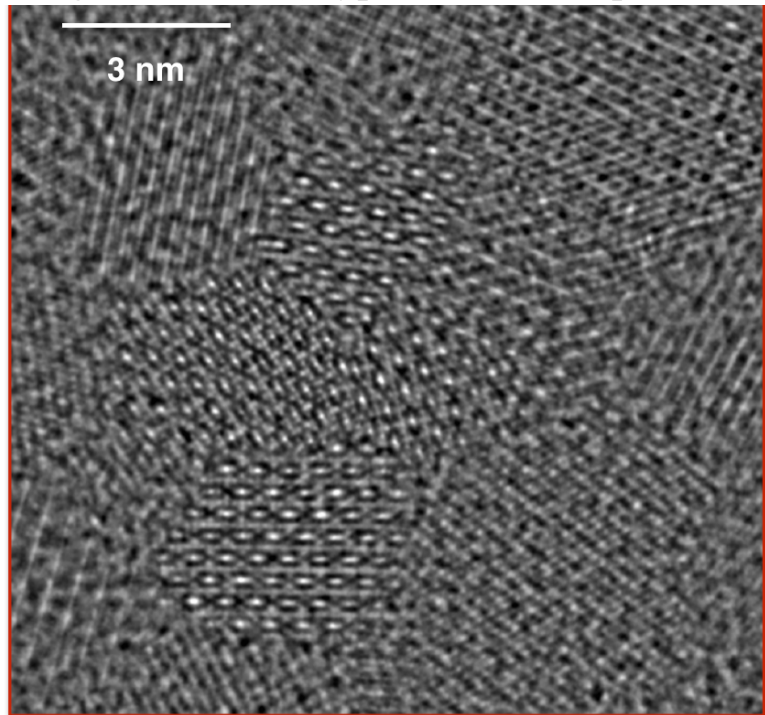

Rate: 1700 e/Å2s

Total: $425200 \mathrm{e} / \AA^{2}$

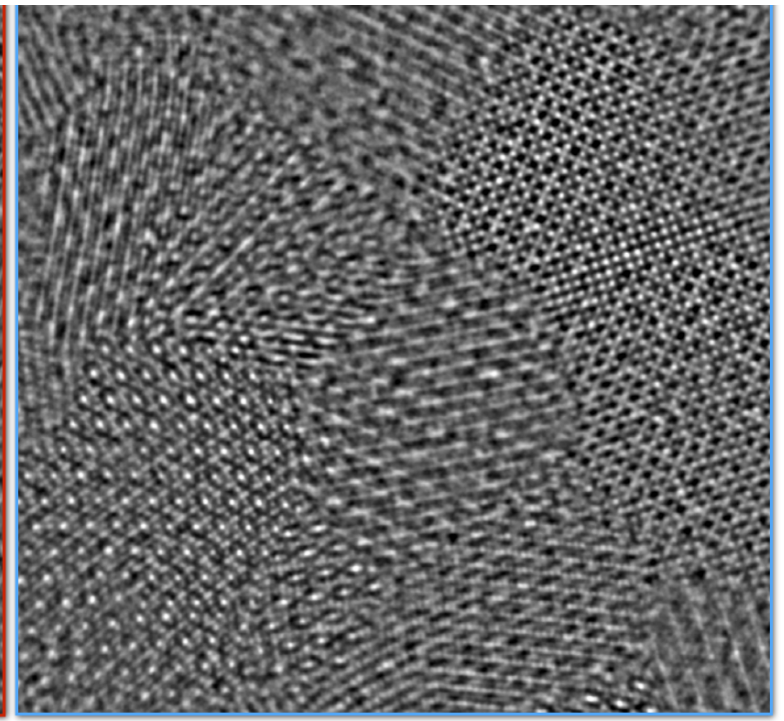

Rate: $5300 \mathrm{e} / \mathrm{A}^{2} \mathrm{~s}$

Total: $1310000 \mathrm{e} / \AA^{2}$

Figure 2. Two successive, reconstructed phase images of a 3-5 nm thick $\mathrm{Co}_{3} \mathrm{O}_{4}$ protection layer that exhibits superior catalytically activity. Grain growth is stimulated by electron beam-induced surface diffusion in interfacial monolayers and Oswald ripening occurs as the accumulated (total) electron dose increases. 